\title{
The Double Chooz reactor neutrino experiment
}

\section{Masaki Ishitsuka ${ }^{* \dagger}$}

Tokyo Institute of Technology

E-mail: ishitsuka@hep.phys.titech.ac.jp

Double Chooz is a reactor neutrino oscillation experiment which aims at discovery of the last neutrino mixing angle, $\theta_{13}$. The expected sensitivity to $\sin ^{2} 2 \theta_{13}$ reaches 0.03 (90\% C.L.) which is approximately a factor 5 better than the current limit. Double Chooz will use two identical detectors with different baselines to suppress the systematic uncertainties down to $1 \%$ or better. The far detector construction is almost completed (as of November 2010) and the physics data taking is expected shortly.

35th International Conference of High Energy Physics

July 22-28, 2010

Paris, France

\footnotetext{
* Speaker.

${ }^{\dagger}$ On behalf of the Double Chooz collaboration
} 


\section{Introduction}

Discovery of neutrino oscillations as a consequence of neutrino mass was a breakthrough to the particle physics beyond the Standard Model. In the Standard Model, neutrinos are all lefthanded particles and have no mass. While the experimental data strongly support the existence of neutrino oscillations [1].

In standard three flavor framework, the oscillation probability is determined by three mixing angles $\left(\theta_{12}, \theta_{23}\right.$ and $\left.\theta_{13}\right)$, three mass squared differences $\left(\Delta m_{21}^{2}, \Delta m_{32}^{2}\right.$ and $\Delta m_{31}^{2}$ in which only two are independent) and one CP-violation phase $\left(\delta_{C P}\right)$. Among three mixing angles, $\theta_{12}$ and $\theta_{23}$ have been measured, while only the limit is given to $\theta_{13}$ by the CHOOZ experiment as $\sin ^{2} 2 \theta_{13}<$ $0.15[2]$. No hint to $\delta_{C P}$ and mass hierarchy in neutrino sector is given yet. Measurement of $\theta_{13}$ is essential to realize the experimental study of $\delta_{C P}$ and mass hierarchy in neutrino oscillations since these can be measured only as combinations with $\theta_{13}$. Therefore, the main target of ongoing or forthcoming neutrino oscillation experiments is to measure the last unknown mixing angle, $\theta_{13}$.

Based on current knowledge, one mass scale is much larger or smaller than the others $\left(\left|\Delta m_{31}^{2}\right| \sim\right.$ $\left.\left|\Delta m_{32}^{2}\right| \gg \Delta m_{21}^{2}\right)$. This allows us to interpret the experimental data by simple two flavor oscillation scheme in many cases. In two flavor scheme, survival probability of $\overline{v_{e}}$ with energy $E_{v}$ after traveling a distance of $L$ is expressed as:

$$
P\left(\overline{v_{e}} \rightarrow \overline{v_{e}}\right)=1-\sin ^{2} 2 \theta_{13} \sin ^{2}\left(\frac{1.27 \Delta m_{31}^{2}\left(\mathrm{eV}^{2}\right) L(\mathrm{~m})}{E_{v}(\mathrm{MeV})}\right) .
$$

This equation is a good approximation to reactor neutrino oscillation even in matter for $L$ less than a few $\mathrm{km} . \theta_{13}$ can be directly measured from the oscillation amplitude in reactor neutrino oscillation.

\section{Experiment Overview}

The Double Chooz experiment is a reactor neutrino oscillation experiment which aims at measurement of $\theta_{13}[3]$. Double Chooz employs two detectors method to reduce systematic errors on the $\theta_{13}$ measurement. Two detectors are installed in the Chooz two-cores $\left(4.27+4.27 \mathrm{GW}_{t h}\right) \mathrm{nu}$ clear power plant in France. Far detector is located at $1.05 \mathrm{~km}$ away from the reactor cores where the oscillation probability becomes maximum for typical reactor neutrino energy (a few MeV). The far detector construction is almost completed and currently liquid scintillator is being filled in the detector (as of November 2010). After liquid scintillator filling is completed by the end of 2010, physics data taking by the far detector is expected shortly. As the detector design is improved and the target volume is enlarged from the $\mathrm{CHOOZ}$ experiment, sensitivity to $\theta_{13}$ reaches the current limit by the $\mathrm{CHOOZ}$ experiment within a few months observation even with the single detector. The near detector will be installed at a distance of $400 \mathrm{~m}$ from reactor cores where the effect of neutrino oscillation is negligible. Construction of the near detector will start immediately after the far detector installation is completed, and the operation is expected in 2012.

\section{Detector}

Reactor neutrinos are detected by delayed coincidence technique by which background events are largely suppressed. Reactor neutrinos interact with free protons through inverse $\beta$-decay reac- 


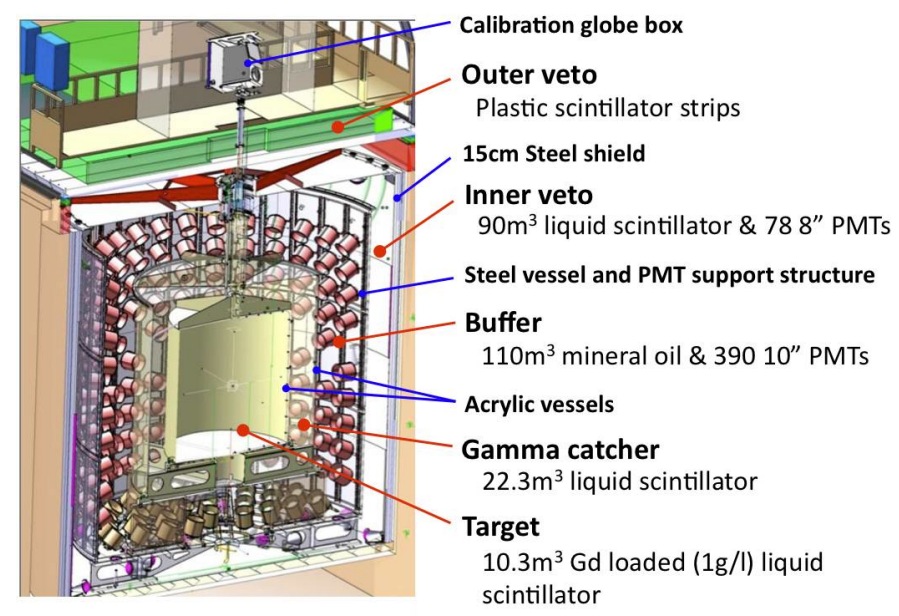

Figure 1: Schematic view of the Double Chooz detector design.

tion: $\overline{v_{e}}+p \rightarrow e^{+}+n$. Positron is observed as the prompt signal and the neutrino energy can be measured from the energy as: $E_{v}=E_{\text {signal }}+0.8 \mathrm{MeV}$. Neutron is captured mainly on gadolinium in liquid scintillator and emit total $8 \mathrm{MeV} \gamma$-rays after $30 \mu \mathrm{sec}$ in average.

Figure 1 shows a schematic view of the Double Chooz detector design. The Double Chooz detector consists of four concentric cylindrical tanks and an outer veto system on the top. The innermost target volume contains $10.3 \mathrm{~m}^{3}$ gadolinium loaded liquid scintillator, surrounded by $55 \mathrm{~cm}$ thick liquid scintillator and $105 \mathrm{~cm}$ thick non-scintillating mineral oil layers. Total $8 \mathrm{MeV} \gamma$-rays are emitted from neutron capture on gadolinium in the target volume, and escaped $\gamma$-rays from the target volume are detected by liquid scintillator layer. The non-scintillating oil layer is called buffer which works as shield to $\gamma$-rays mainly from radioactivity of PMTs. Introduction of the buffer volume is one of the major improvements with respect to the $\mathrm{CHOOZ}$ detector. 390 low background 10-inch PMTs are installed in the buffer tank to collect scintillation light from the inner volumes. These three regions and PMTs constitute the central detector system referred to as inner detector and optically separated from the outer layer.

The inner detector is surrounded by $50 \mathrm{~cm}$ thick liquid scintillator layer called inner veto. The inner veto works not only as cosmic ray muon veto but also as shield to fast neutrons from outside of the detector. The whole detector is further surrounded by $15 \mathrm{~cm}$ thick steel shield and protected against $\gamma$-rays. In addition, outer veto plastic scintillator strips will be installed on the top of the main detector to identify cosmic ray muons.

\section{Prospects of $\theta_{13}$ measurement}

Significant improvements in both statistical and systematic errors are expected in the Double Chooz experiment with respect to the $\mathrm{CHOOZ}$ experiment. With enlarged target volume and newly developed gadolinium loaded liquid scintillator with improved stability, statistical error will be reduced to less than $0.5 \%$ after three years of data taking. Major systematic error sources in the $\mathrm{CHOOZ}$ experiment were the uncertainties on reactor neutrino flux, number of target protons and 


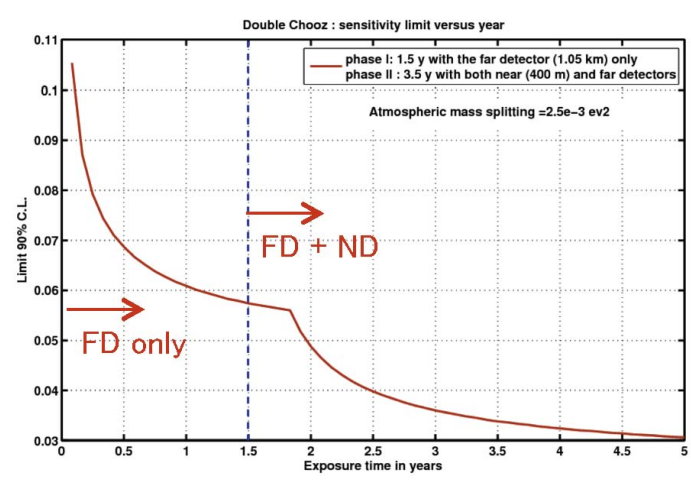

Figure 2: Expected $90 \%$ C.L. limit to $\sin ^{2} 2 \theta_{13}$ (for $\left|\Delta m_{31}^{2}\right|=2.5 \times 10^{-3} \mathrm{eV}^{2}$ ) as a function of time.

selection efficiency. Uncertainty on neutrino flux is canceled by comparing measurements by two detectors at different baselines. Double Chooz will construct two detectors based on the same detector design by which systematic uncertainties on the number of free protons in the target volume and selection efficiency are canceled in comparison of two measurements. In order to make two detectors essentially identical, detector components including liquid scintillator are made simultaneously for both detectors. Total systematic error will be reduced to less than $1 \%$ in the Double Chooz experiment.

Figure 2 shows the expected sensitivity to $\sin ^{2} 2 \theta_{13}$ assuming the near detector operation will start 1.5 years after the far detector operation started. After 1.5 years of data taking by the far detector, the sensitivity to $\sin ^{2} 2 \theta_{13}$ reaches 0.06 at $90 \%$ C.L.. In the second phase with near and far detectors, the systematic error is largely reduced. The sensitivity reaches 0.03 in 3 years operation with two detectors, which is a factor 5 better than the current limit.

\section{Conclusion}

The Double Chooz far detector construction is almost completed (as of November 2010) and the physics data taking is expected shortly. The near detector operation is expected to start in 2012. The expected sensitivity to $\sin ^{2} 2 \theta_{13}$ reaches 0.06 in 1.5 years of data taking with the single detector and 0.03 in three years with two detectors.

\section{Acknowledgement}

I acknowledge financial support from the Global Center of Excellence Program by MEXT, Japan through the Nanoscience and Quantum Physics Project of the Tokyo Institute of Technology.

\section{References}

[1] K. Nakamura et al., J. Phys. G37, 075021 (2010).

[2] M. Apollonio et al., Eur. Phys. J. C27, 331-374 (2003).

[3] F. Ardellier et al., arXiv:hep-ex/0606025 (2006). 